\title{
Alteration of spontaneous emission in hydrogenated amorphous silicon nitride microcavities
}

\author{
Ali Serpengüzel *, Atilla Aydinli, Alpan Bek \\ Bilkent University, Physics Department, Bilkent, Ankara 06533, Turkey
}

\begin{abstract}
A Fabry-Perot microcavity is used for the alteration of the spontaneous emission spectrum in hydrogenated amorphous silicon nitride. The modified photon density of states of the Fabry-Perot microcavity are responsible for the alteration of the spontaneous emission spectrum. The Fabry-Perot microcavity enhances the intensity of the spontaneous emission signal by a factor of 4 at the photon energies corresponding to the microcavity resonances. The $0.075 \mathrm{eV}$ wide spontaneous emission linewidth of the Fabry-Perot microcavity resonances is 7 times smaller than the $0.5 \mathrm{eV}$ wide spontaneous emission linewidth of the bulk hydrogenated amorphous silicon nitride. (c) 1998 Elsevier Science B.V. All rights reserved.
\end{abstract}

PACS: 71.23.Cq; 73.61.Jc; 78.66.Jg; 78.55.-m; 78.30.-j

Keywords: Fabry-Perot microcavity; Spontaneous emission spectrum; Hydrogenated amorphous silicon nitride

\section{Introduction}

There has been a lot of progress made in the study of the modification of the spontaneous emission (SE) in planar microcavities in a number of organic [1] and inorganic [2] semiconductors. In a planar microcavity, electromagnetic and quantum electrodynamic effects occur. First, the microcavity acts as an optical resonator for photons with specific energies, which after one round trip, return to their starting position in phase. Second, the SE cross-sections at the microcavity resonance photon energies are larger than the bulk SE cross-sections because of the modified photon density of states of the microcavity [3]. For the

\footnotetext{
* Corresponding author. Tel.: +90-312 2664000 ext. 1965; fax: +90-312 266 4579; e-mail: serpen@fen.bilkent.edu.tr.
}

same reason, the SE cross-sections in between the cavity resonances are smaller than the bulk SE cross-sections. In a planar microcavity, the photon states are the cavity resonances, while for bulk samples, the photon states are the continuum modes of an infinite system [4,5]. Planar microcavity effects on the $\mathrm{SE}$ of porous silicon [6], and $\mathrm{Si} / \mathrm{SiO}_{x}$ superlattices [7] have been reported.

There is a great deal of interest in silicon as a potential optoelectronic material. With modern process techniques, it will be possible to integrate lasers, photodetectors and waveguides on optoelectronic silicon motherboards. Hydrogenated amorphous silicon (a-Si:H) has been used for the realization of planar waveguides, $[8,9]$ which will be able to route optical signals within such silicon motherboards. a-Si:H can be deposited by plasma-enhanced chemical vapor deposition (PECVD) on almost any substrate at tem- 
peratures below $500 \mathrm{~K}$, which makes it compatible with the microelectronic technology. a-Si:H [10], porous silicon [11], and other silicon based materials [12] also exhibit high external quantum efficiency electroluminescence and photoluminescence at room temperature. These properties make silicon-based materials potential candidates for novel optical gain media.

Recently, we have observed visible SE from a$\mathrm{Si}: \mathrm{H}$, as well as its oxides and nitrides $\left(\mathrm{a}-\mathrm{SiN}_{x}: \mathrm{H}\right)$ grown by low temperature PECVD. [13,14]. In this paper, we report the alteration of the $\mathrm{SE}$ in an a-SiN ${ }_{x}: \mathrm{H}$ Fabry-Perot microcavity.

\section{Experimental procedures}

In our samples, the planar microcavity was realized by a gold $(\mathrm{Au})$ back mirror and an a-SiN $\mathrm{N}_{x}: \mathrm{H}$-air interface front mirror. First, the thin glass substrates were coated with Au. Second, a thin layer of a$\mathrm{SiN}_{x}: \mathrm{H}$ was deposited on the $\mathrm{Au}$ coated substrates by PECVD at $373 \mathrm{~K}$ with a gaseous mixture of $98 \%$ $\mathrm{N}_{2}$ and $2 \% \mathrm{SiH}_{4}$. The flow rate of the gaseous mixture was $180 \mathrm{sccm}$, the radio frequency (RF) power $10 \mathrm{~W}$, and the deposition chamber pressure 1 Torr. The metric thickness $(L)$ of the a-SiN ${ }_{x}: \mathrm{H}$ layer was measured with a surface texture analysis system (Veeco Dektak $3030 \mathrm{ST}$ ) to be $1.40 \pm 0.05 \mu \mathrm{m}$. For reference, another microcavity without the Au back mirror was also fabricated by depositing only a$\mathrm{SiN}_{x}: \mathrm{H}$ on the glass substrates. Hereafter, the microcavity with the Au back mirror will be referred as the metallic microcavity (MM), while the reference microcavity without the $\mathrm{Au}$ back mirror will be referred to as the dielectric microcavity (DM).

The SE spectra were measured with a 1-m double spectrometer (Jobin-Yvon U1000) with a resolution of $0.001 \mathrm{eV}$ and whose exit slit was equipped with a GaAs photomultiplier tube (PMT). The SE spectra were later corrected for the responsivity of the spectrometer and the photomultiplier tube. $\mathrm{An} \mathrm{Ar}^{+}$laser with a photon energy of $2.41 \mathrm{eV}$ and a power of 420 $\mathrm{mW}$ was focused with a $15-\mathrm{cm}$ focal length cylindrical lens on the samples. The SE spectra were taken at $0^{\circ}$ with the laser at $30^{\circ}$ with respect to the surface normal. The reflectance spectra were measured at $0^{\circ}$ with respect to the surface normal using a Varian
Cary 5 spectrophotometer with a resolution of 0.01 $\mathrm{eV}$.

While the exact mechanism of the occurrence of the $\mathrm{SE}$ in bulk a-SiN $x$ :H is still under discussion, we have suggested in Ref. [14] the use of the quantum confinement model [15]. There, it was proposed that our samples consist of small a-Si clusters in a matrix of $\mathrm{a}-\mathrm{SiN}_{x}: \mathrm{H}$. The regions with $\mathrm{Si}-\mathrm{H}$ and $\mathrm{Si}-\mathrm{N}$, having larger energy gaps due to strong $\mathrm{Si}-\mathrm{H}$ and $\mathrm{Si}-\mathrm{N}$ bonds, isolate these a-Si clusters, and form barrier regions around them. The SE originates from these a-Si clusters.

\section{Results}

The reflectance and SE spectra show Fabry-Perot resonances at photon energies $\left(E_{\mathrm{m}}\right)$ satisfying the microcavity resonance condition:

$E_{m}=\frac{h c m}{2 L n}$

where $h$ is the Planck constant, $c$ the speed of light, $m$ the quantized mode number, $L$ the metric thickness, and $n$ the refractive index of the microcavity.

Fig. 1 shows the experimentally measured reflectance spectrum of the DM. The metric thickness of the DM is found to be $L=1.376 \mu \mathrm{m}$ from the spacing of the reflectance dips, which correspond to the Fabry-Perot resonances. This value agrees well with the measured thickness of $1.40 \pm 0.05 \mu \mathrm{m}$ for a refractive index value of $n=2.1$. The mode numbers of the Fabry Perot resonances were found to range

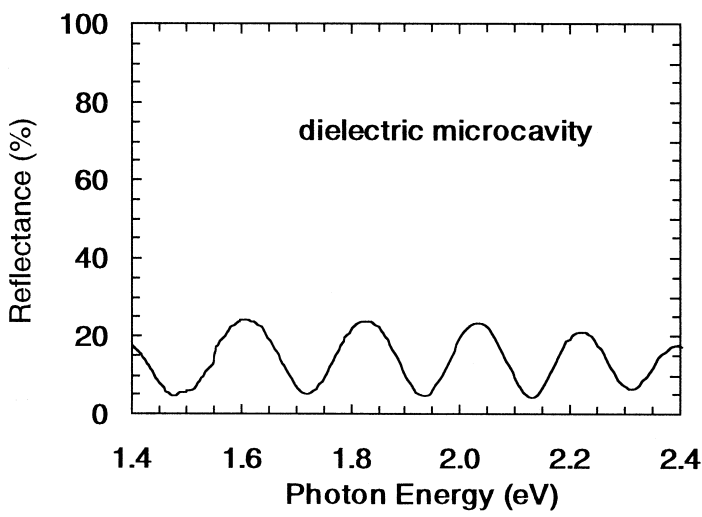

Fig. 1. Reflectance spectrum of the dielectric microcavity. 


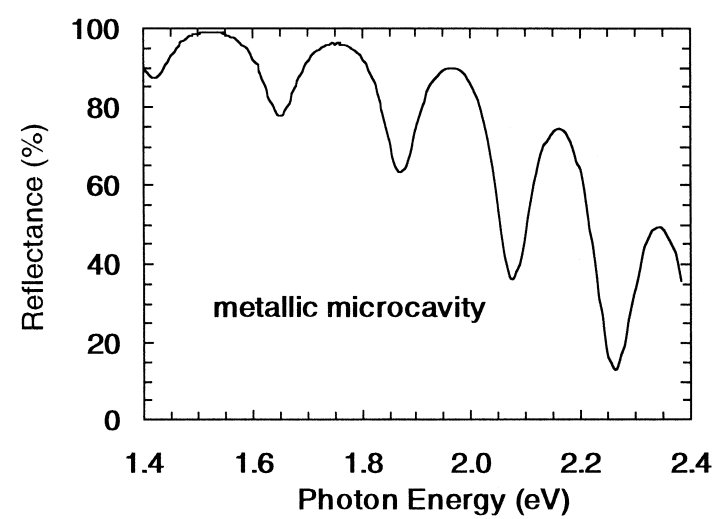

Fig. 2. Reflectance spectrum of the metallic microcavity.

from $m=7\left(E_{7}=1.49 \mathrm{eV}\right)$ to $m=11\left(E_{11}=2.31\right.$ $\mathrm{eV})$. These Fabry-Perot resonances have quality factors of $Q=20$. The decrease of the reflectance towards higher photon energies is due to the absorption of the a-SiN $: \mathrm{H}$.

Fig. 2 depicts the reflectance spectrum of the MM. The metric thickness of the MM was found to be $L=1.438 \mu \mathrm{m}$ from the spacing of the reflectance dips, which correspond to the Fabry-Perot resonances. This value agrees well with the experimental thickness of $1.40 \pm 0.05 \mu \mathrm{m}$ for a refractive index of $n=2.1$. The Fabry-Perot resonances have a quality factor of $Q=30$. The mode numbers of these resonances were found to range from $m=7\left(E_{7}=1.42\right.$ $\mathrm{eV})$ to $m=11\left(E_{11}=2.26 \mathrm{eV}\right)$. The decrease of the reflectance towards higher photon energies is due to

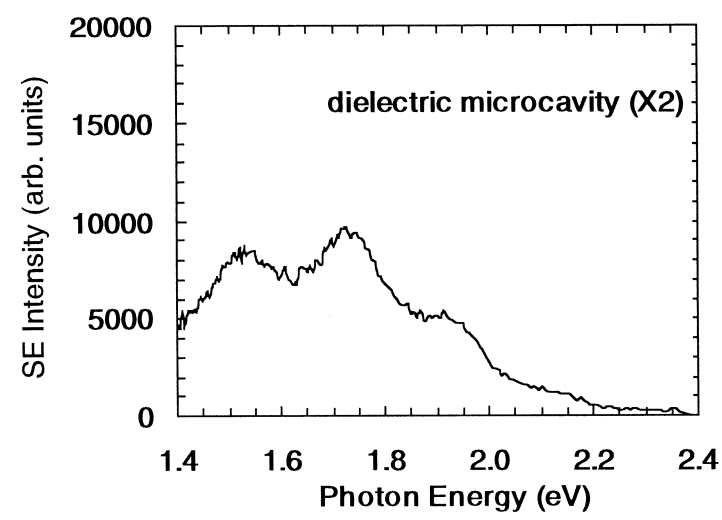

Fig. 3. Spontaneous emission spectrum of the dielectric microcavity.

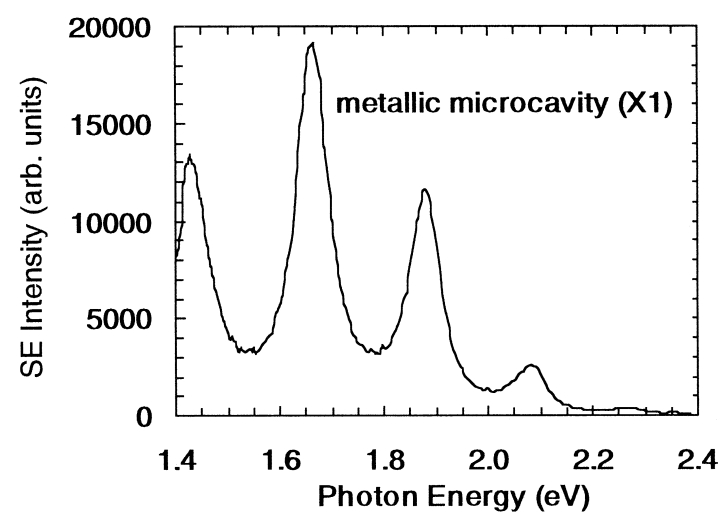

Fig. 4. Spontaneous emission spectrum of the metallic microcavity.

the absorption of the $\mathrm{a}-\mathrm{SiN}_{x}: \mathrm{H}$ and the $\mathrm{Au}$ back mirror.

Fig. 3 shows the SE spectrum of the DM. The SE is modulated by the weak Fabry-Perot resonances, which correlate well with the reflectance minima of Fig. 1. Above $E=2 \mathrm{eV}$, the resonances start to wash out by the absorption of the a-SiN ${ }_{x}: \mathrm{H}$. The loading of the resonances by the a-SiN $\mathrm{S}_{x}: \mathrm{H}$ absorption stops below $E=2 \mathrm{eV}$.

The SE of a-SiN ${ }_{x}: \mathrm{H}$ has a broad linewidth $(\mathrm{FWHM}=0.5 \mathrm{eV})$ and an external peak efficiency of $3 \%$, both of which correlate well with the values measured for a-Si:H [16]. While the external SE peak efficiency of a- $\mathrm{SiN}_{x}: \mathrm{H}$ is 3 times lower than that of GaAs $(10 \%)$, the linewidth of a-SiN $x: \mathrm{H}(\mathrm{FWHM}=$ $0.5 \mathrm{eV}$ ) is 5 times greater than the linewidth of GaAs $(\mathrm{FWHM}=0.1 \mathrm{eV})$. The external SE efficiency and linewidth of the a-SiN $: \mathrm{H}$ show that, $\mathrm{a}-\mathrm{SiN}_{x}: \mathrm{H}$ has potential as a novel photonic material.

Fig. 4 shows the SE spectrum of the MM. The SE is modulated by the strong Fabry-Perot resonances, which correlate well with the reflectance minima of Fig. 2. Above $E=2 \mathrm{eV}$, the resonances start to wash out by the absorption of the a-SiN $: \mathrm{H}$ and $\mathrm{Au}$. The loading of the resonances by the a-SiN $x: \mathrm{H}$ and $\mathrm{Au}$ absorption stops below $E=2 \mathrm{eV}$.

\section{Discussion}

The two SE spectra of Figs. 3 and 4 were obtained under the same experimental conditions. The 
SE spectrum of the DM in Fig. 3 was multiplied by a factor of 2, in order to compare it with the SE spectrum of the MM in Fig. 4. When comparing the spectra in Figs. 3 and 4, the SE of the MM has several noteworthy features with respect to the SE of the DM: (1) there is a 2.2 times increase of the overall spectrum average (i.e., averaging out the Fabry-Perot resonances), (2) there is a 4 times enhancement of the SE peaks, and (3) the SE dips have similar amplitude in the unmultiplied spectra.

The Au mirror reflects the SE light that would otherwise be lost from the back surface and increases the SE by a factor of 2. The reflection of the excitation laser from the Au mirror also increases the SE by a factor of 1.2, since the metric thickness $1.438 \mu \mathrm{m}$ of the $\mathrm{MM}$ is shorter than the a-SiN $x: \mathrm{H}$ absorption depth of $2 \mu \mathrm{m}$ (corresponding to the measured absorption coefficient of $0.5 \mu \mathrm{m}^{-1}$ ) at the $\mathrm{Ar}^{+}$laser photon energy. Therefore, the expected increase of the overall spectrum average is 2.4, which is the multiplication of both factors and comparable with the measured value of 2.2. The 4 times enhancement at the resonances, is due to the combined effect of the enhancement of the SE by the resonances with that of the SE and the laser light reflecting from the Au mirror. The SE dips having the same amplitude in both spectra is due to the inhibition of the SE in between the resonances.

\section{Conclusions}

In conclusion, a planar microcavity is used for the alteration of $\mathrm{SE}$ in $\mathrm{a}-\mathrm{SiN}_{x}: \mathrm{H}$. The alteration of the SE is understood by the microcavity modified photon density of states. The microcavity with a $\mathrm{Au}$ back mirror enhances the SE signal by a factor of 4 at the resonant photon energies. The SE linewidth $(0.075 \mathrm{eV})$ of the microcavity resonances is 7 times smaller than the SE linewidth $(0.5 \mathrm{eV})$ of the a$\mathrm{SiN}_{x}: \mathrm{H}$. The microcavity alteration of the $\mathrm{SE}$ in
a-SiN $\mathrm{N}_{x}: \mathrm{H}$ opens up the way for a novel generation of resonant cavity enhanced optoelectronic light emitting devices.

\section{Acknowledgements}

We would like to thank M. Güre for his help with the growth of the samples. We would like to acknowledge the support of this research by the Scientific and Technical Research Council of Turkey Grants TBAG-1368 and 1244 and the International Center for Theoretical Physics Grant 95-500 RG/PHYS/AS.

\section{References}

[1] A. Dodabalapur, L.J. Rothberg, R.H. Jordan, T.M. Miller, R.E. Slusher, J.M. Phillips, J. Appl. Phys. 80 (1996) 6954.

[2] H. Yokoyama, Science 256 (1992) 66.

[3] E.M. Purcell, Phys. Rev. 69 (1946) 681.

[4] F. De Martini, G. Innocenti, G.R. Jacobowitz, P. Mataloni, Phys. Rev. Lett. 59 (1987) 2955.

[5] H. Yokoyama, K. Nishi, T. Anan, H. Yamada, S.D. Brorson, E.P. Ippen, Appl. Phys. Lett. 57 (1990) 2814.

[6] L. Pavesi, R. Guardini, C. Mazolleni, Solid State Commun. 97 (1996) 1051.

[7] B.T. Sullivan, D.J. Lockwood, H.J. Labbe, Z.-H. Lu, Appl. Phys. Lett. 69 (1996) 3149.

[8] A.M. Agarwal, L. Liao, J.S. Foresi, M.R. Black, X. Duan, L.C. Kimerling, J. Appl. Phys. 80 (1996) 6120.

[9] G. Cocorullo, F.G. Della Corte, I. Rendina, C. Minarini, A. Rubino, E. Terzini, Opt. Lett. 21 (1996) 2002.

[10] D.J. Lockwood, Solid State Commun. 92 (1994) 101.

[11] T. Canham, Appl. Phys. Lett. 57 (1990) 1046.

[12] W.B. Pollard, G. Lucovsky, Phys. Rev. B 26 (1982) 3172.

[13] F.N. Timofeev, A. Aydinli, R. Ellialtioglu, K. Türkoglu, M. Güre, V.N. Mikhailov, O.A. Lavrova, Solid State Commun. 95 (1995) 443

[14] A. Aydinli, A. Serpengüzel, D. Vardar, Solid State Commun. 98 (1996) 273.

[15] M.H. Brodsky, Solid State Commun. 36 (1980) 55.

[16] G.A.N. Connell, in: M.H. Brodsky (Ed.), Amorphous Semiconductors, Springer-Verlag, Berlin, 1985, p. 73. 